\title{
An exploration on the influence of positive simple thyroid peroxidase antibody on female infertility
}

\author{
XIANPING WANG $^{1 *}$, XUFENG DING $^{2 *}, \mathrm{XIAO} \mathrm{XIAO}^{3}$, FANG XIONG $^{3}$ and RUI FANG ${ }^{3}$ \\ ${ }^{1}$ Department of Gynaecology and Obstetrics, The Affiliated Wuxi Maternity and Child Health Care Hospital of \\ Nanjing Medical University, Wuxi, Jiangsu 214400; ${ }^{2}$ Department of Reproductive Health, Maternal and \\ Child Health Care Hospital of Yixing, Wuxi, Jiangsu 214200; ${ }^{3}$ Reproductive Medicine Center, \\ Maternal and Child Health Care Hospital of Wuxi, Wuxi, Jiangsu 214002, P.R. China
}

Received September 22, 2017; Accepted July 20, 2018

DOI: $10.3892 / \mathrm{etm} .2018 .6561$

\begin{abstract}
The aim of the present study was to explore the influence of positive simple thyroid peroxidase antibody (TPO-Ab) on female infertility. Venous blood was collected on an empty stomach from infertile female patients (all of whom were in line with the World Health Organization's diagnostic criteria for infertility) receiving treatments at The Affiliated Wuxi Maternity and Child Health Care Hospital of Nanjing Medical University from 2014 to 2017 to detect thyroid function and thyroid antibodies [thyroglobulin antibody (TG-Ab), TPO-Ab and thyroid microsome antibody (TM-Ab)]. A total of 529 patients with normal thyroid function, TG-Ab and $\mathrm{TM}-\mathrm{Ab}$ were included in the present study; they were divided into the positive group (121 cases with positive TPO-Ab) and the negative group (408 cases with negative TPO-Ab). Comparisons of age, body mass index (BMI), basal hormone levels, irregular menstruation, tubal obstruction, premature ovarian failure (POF), endometriosis (EMT) and polycystic ovary syndrome (PCOS) between the two groups of patients were conducted in order to determine whether the differences were statistically significant. The incidence rate of EMT was $39.6 \%$ in the positive group and $17.1 \%$ in the negative group $(\mathrm{P}<0.05)$. The incidence rate of PCOS in the observation group was $43.9 \%$, which was significantly higher than that of the control group $(21.3 \%$; $\mathrm{P}<0.01)$. The results revealed that the differences in age, BMI, basal hormone levels, irregular menstruation and POF between the two groups were not statistically significant $(\mathrm{P}>0.05)$. Finally, the factors that had
\end{abstract}

Correspondence to: Ms. Xiao Xiao, Reproductive Medicine Center, Maternal and Child Health Care Hospital of Wuxi, 48 Huaishu Lane, Wuxi, Jiangsu 214002, P.R. China

E-mail: xbz4w5@163.com

*Contributed equally

Key words: positive simple thyroid peroxidase antibody, thyroid autoantibodies, endometriosis, polycystic ovary syndrome, female infertility statistical significance (EMT and PCOS) were stratified by different age groups to compare the incidence rate of positive results among the different age groups, thus concluding which age group was influenced to a greater extent by TPO-Ab. The results indicated that positive TPO-Ab may be associated with PCOS and EMT, though particularly with PCOS. Infertile PCOS patients aged 28-35 years old were influenced more by $\mathrm{TPO}-\mathrm{Ab}$ than those in other age groups. Thus, it is recommended that thyroid autoantibodies are detected in infertile PCOS females aged 28-35 years old, and treatment should be administered as soon thereafter.

\section{Introduction}

Infertility is currently a common disease in women at childbearing age. With the acceleration of social processes, the morbidity of infertility is increased gradually throughout the world at present. In developed countries, $10-15 \%$ of couples suffer from infertility (1). Common causes of infertility include factors of male infertility, female infertility, infertility in couples and unexplained infertility (2-4), among which factors of female infertility take up the most. Meanwhile, female infertility is caused by the following definite reasons: Immune infertility, tubal obstruction and ovulatory dysfunction $(5,6)$. Zhong et al (7), showed that in vitro fertilization rates and embryo implantation rate was significantly reduced in anti-thyroid antibody-positive patients. At present, the number of infertile women in China is increased year by year, which requires us to find more effective influence indicators to judge and find out the causes of infertility in a better way.

\section{Materials and methods}

General data. Objects of study: A total of 529 infertile females that received treatments in The Affiliated Wuxi Maternity and Child Health Care Hospital of Nanjing Medical University from January 2014 to January 2017 and complied with inclusion criteria were selected for retrospective study. They were divided into positive group (121 patients) and negative group (408 patients). The patients were $22-43$ years with an average age of $30.5 \pm 5.9$. General data of the patients, including age, height, body weight, initial menstruation, duration of 
Table I. General data of 529 infertile female patients.

\begin{tabular}{lccr}
\hline Variables & Positive group $(\mathrm{n}=121)$ & Negative group $(\mathrm{n}=408)$ & P-value \\
\hline Age (years) & $29.75 \pm 5.21$ & $30.83 \pm 6.23$ & 0.626 \\
Height (m) & $1.59 \pm 0.07$ & $1.60 \pm 0.08$ & 0.875 \\
Weight (kg) & $57.20 \pm 10.70$ & $54.96 \pm 9.61$ & 0.332 \\
Initial menstruation (years) & $13.80 \pm 2.80$ & $14.16 \pm 3.12$ & 0.672 \\
Duration of infertility (years) & $3.70 \pm 2.81$ & $4.21 \pm 3.10$ & 0.552 \\
Menstrual cycle (days) & $27.95 \pm 13.21$ & $28.21 \pm 14.43$ & 0.745 \\
Permanent residence (city/village) $_{\text {Education background (above/under) }}{ }^{\mathrm{a}}, \mathrm{n}$ & $79 / 42$ & $272 / 136$ & 0.778 \\
& $69 / 52$ & $229 / 179$ & 0.861
\end{tabular}

aWhere 'above' refers to a university degree or higher qualification, and 'under' refers to qualifications below a university degree.

infertility, menstrual cycle, permanent residence (city/village) and education background (university degree or above/under university degree) are given in Table I. The present study was conducted in accordance with the Declaration of Helsinki and was approved by the Ethics Committee of The Affiliated Wuxi Maternity and Child Health Care Hospital of Nanjing Medical University (Wuxi, China). All the participants or their family members signed written informed consent.

Inclusion criteria: i) Married women at childbearing age (20-49 years old); ii) patients complying with World Health Organization (WHO) diagnostic criteria for female infertility: females with sexual life; females without pregnancy or abortion; females without pregnancy while no contraceptive measure was taken within 1 year; iii) patients with normal thyroid function and thyroid autoantibodies [thyroglobulin antibody (TG-Ab), thyroid peroxidase antibody (TPO-Ab) and thyroid microsome antibody (TM-Ab)]; iv) patients with positive TPO-Ab (a thyroid autoantibody) and normal TG-Ab and TM-Ab. Patients meeting item i, ii and iii were grouped into negative control group (negative group for short), and those meeting item $\mathrm{i}$, ii and iv into positive simple TPO-Ab group (positive group for short).

Exclusion criteria: i) patients with a history of diabetes, coronary heart disease, hyperlipidemia, hypertension, diseases caused by hypothalamic or pituitary dysfunction, malignant tumors, substantial lesions of organs such as liver and kidney, etc.; and ii) patients with a history of thyroid diseases (whether or not cured), patients with recent oral administration of drugs that can affect thyroid function and blood lipid levels, patients with a history of thyroid antibody positive.

Laboratory examinations. Venous blood was collected from all the included infertile females on an empty stomach in the morning from the 2 nd day to the 4 th day after menstruation to measure basal hormone levels. The levels of human follicle stimulating hormone (hFSH), estradiol (ESTRDL), progesterone (Prog), prolactin (PRL), testosterone (TESTO) and human luteinizing hormone (hLH) in the serum were measured by chemiluminescence method (Beijing Craymei Biotech Co., Ltd., Beijing, China). All the items of blood drawing were conducted by professional nurses, and relevant tests and results were completed and judged, respectively by two specific laboratory physicians.
Table II. Comparisons of BMI and basal hormone levels in the serum between the two groups.

\begin{tabular}{lccc}
\hline Variables & Positive group & Negative group & P-value \\
\hline Age $($ years $)$ & $29.75 \pm 5.21$ & $30.83 \pm 6.23$ & 0.626 \\
BMI $\left(\mathrm{kg} / \mathrm{m}^{2}\right)$ & $24.68 \pm 3.67$ & $24.21 \pm 3.82$ & 0.782 \\
$\mathrm{hFSH}(\mathrm{mIU} / \mathrm{ml})$ & $7.56 \pm 2.20$ & $7.23 \pm 1.96$ & 0.725 \\
ESTRDL $(\mathrm{pg} / \mathrm{ml})$ & $36.20 \pm 21.43$ & $37.76 \pm 28.51$ & 0.631 \\
Prog $(\mathrm{ng} / \mathrm{ml})$ & $0.79 \pm 0.63$ & $0.82 \pm 0.58$ & 0.599 \\
PRL $(\mathrm{ng} / \mathrm{ml})$ & $14.92 \pm 8.24$ & $14.88 \pm 9.01$ & 0.838 \\
TESTO $(\mathrm{ng} / \mathrm{dl})$ & $44.53 \pm 19.25$ & $47.79 \pm 21.98$ & 0.721 \\
$\mathrm{hLH}(\mathrm{mIU} / \mathrm{ml})$ & $3.29 \pm 1.96$ & $3.38 \pm 2.11$ & 0.824 \\
\hline
\end{tabular}

BMI, body mass index; hFSH, follicle stimulating hormone; ESTRDL, estradiol; Prog, progesterone; PRL, prolactin; TESTO, testosterone; hLH, human luteinizing hormone.

Confirmation on causes of infertility. The causes of female infertility were determined and studied by inquiry of medical history, general gynecological examination, detection of basal hormone levels, B-scan ultrasonography, tubal and uterine lipiodol angiography (8), laparoscopy, hysteroscopy, surgical pathologic examination and other ways. In addition, polycystic ovarian syndrome (PCOS) was diagnosed as per industry standard of the Ministry of Health (2011).

Comparisons of relative indicators among groups. The correlation of positive simple TPO-Ab with the diseases of infertility such as endometriosis (EMT), PCOS, tubal obstruction and premature ovarian failure (POF) was compared. All the patients were divided into three age groups (22-28, 28-35 and 35-42 years old) to analyze and compare the correlation of infertility with positive simple TOP-Ab.

Statistical analysis. All the data were processed using SPSS v.20.0 software (IBM Corp., Armonk, NY, USA). The measurement data were expressed as the mean \pm standard deviation. Data between the two groups was compared by t-test. Enumeration data was expressed as percentages, and the Chi-square test was performed for data analysis. $\mathrm{P}<0.05$ 
Table III. Comparison of incidence rates of diseases of infertility among 529 infertile females.

\begin{tabular}{|c|c|c|c|c|c|c|}
\hline \multirow[b]{2}{*}{ Disease } & \multicolumn{2}{|c|}{ Positive group $(\mathrm{n}=121)$} & \multicolumn{2}{|c|}{ Negative group $(n=408)$} & \multirow[b]{2}{*}{$\chi^{2}$} & \multirow[b]{2}{*}{ P-value } \\
\hline & $\mathrm{n}$ & $\%$ & $\mathrm{n}$ & $\%$ & & \\
\hline Irregular menstruation & 25 & 20.7 & 65 & 15.9 & 1.479 & 0.224 \\
\hline Tubal obstruction & 19 & 15.7 & 89 & 21.8 & 2.145 & 0.143 \\
\hline EMT & 34 & 28.1 & 76 & 18.6 & 5.083 & 0.024 \\
\hline POF & 12 & 9.9 & 43 & 10.5 & 0.039 & 0.844 \\
\hline PCOS & 45 & 37.1 & 79 & 19.4 & 16.527 & $<0.001$ \\
\hline
\end{tabular}

EMT, endometriosis; PCOS, polycystic ovary syndrome; POF, premature ovarian failure.

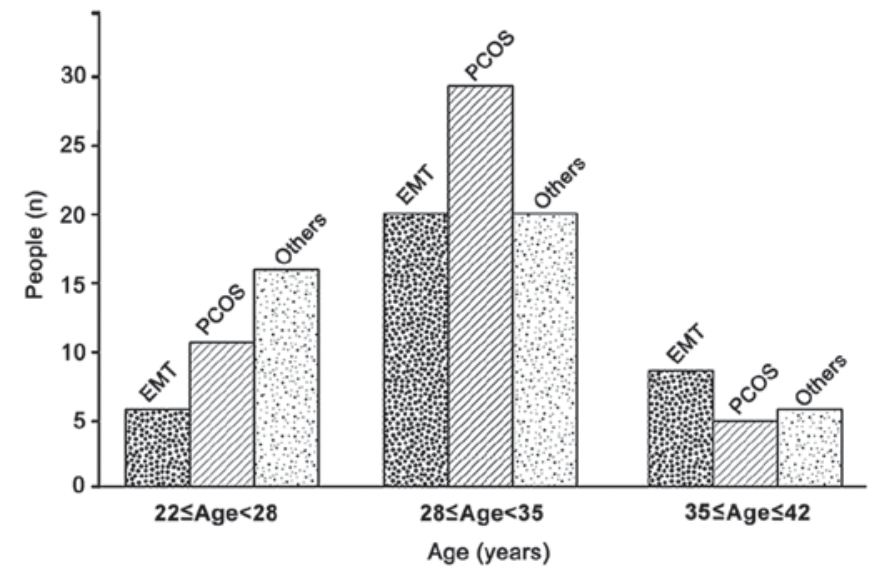

Figure 1. Comparisons between the number of patients with EMT and PCOS among the different age groups. The majority of the two groups were aged 28-35 years old. Patients aged 28-35 years old represented a relatively high percentage of PCOS cases, while those aged 35-42 years old accounted for the largest percentage in EMT cases. EMT, endometriosis; PCOS, polycystic ovary syndrome.

was considered to indicate a statistically significant difference.

\section{Results}

Comparisons of BMI and basal hormone levels in the serum between the two groups. A total of 529 infertile females were included in this study. The differences in body mass index (BMI) and basal hormone levels (hFSH, ESTRD, Prog, PRL, TESTO and hLH) had no statistical significance $(\mathrm{P}>0.05$; Table II).

Comparison of incidence rates of diseases of infertility between the two groups. The comparison of menstruation between the two groups of patients showed no statistical significance $(\mathrm{P}>0.05)$. The incidence rates of tubal obstruction and $\mathrm{POF}$ in the positive group were lower than those in the negative group, but the differences between the two groups were not significant $(\mathrm{P}>0.05)$. However, the differences in the incidence rates of EMT and PCOS between the two groups of females were significant $(\mathrm{P}<0.05)$, and the incidence rate of PCOS was relatively significant in females with positive simple TPO-Ab $(\mathrm{P}<0.001$; Table III).

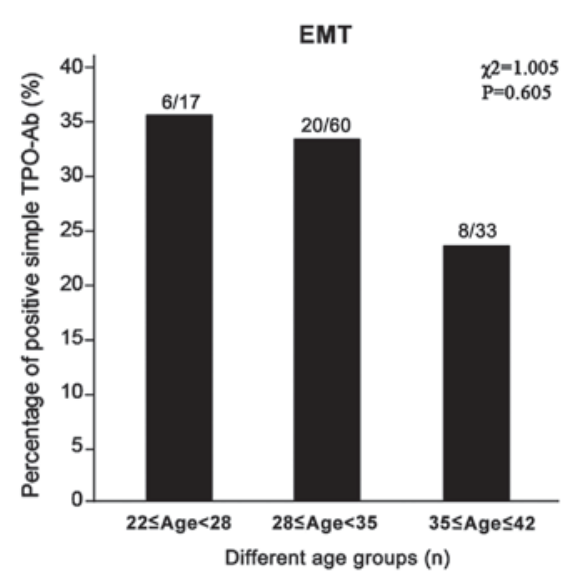

Figure 2. Comparison between the percentage of positive simple TPO-Ab among the different age groups of patients with EMT. The comparison of percentage of positive simple TPO-Ab among the three age groups was not statistically significant $\left(\chi^{2}=1.005 ; \mathrm{P}=0.605\right)$. TPO-Ab, thyroid peroxidase antibody; EMT, endometriosis.

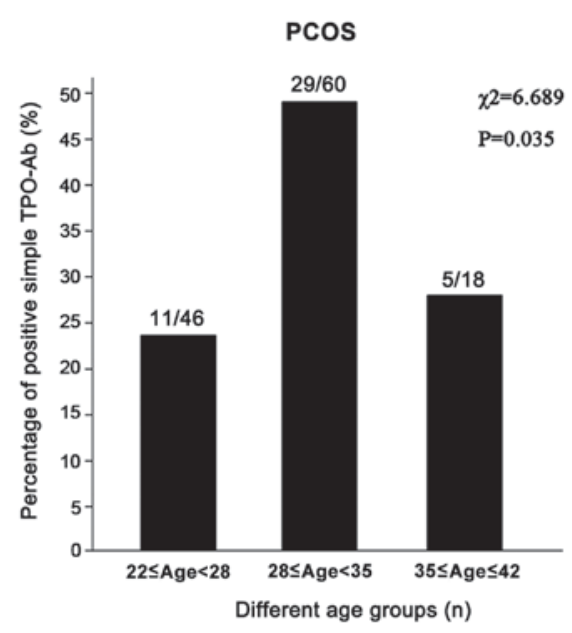

Figure 3. Comparison between the percentage of positive simple TPO-Ab among the different age groups of patients with PCOS. The percentage of positive simple TPO-Ab was relatively high in patients aged 28-35 years, and the difference was statistically significant $\left(\chi^{2}=6.689 ; \mathrm{P}=0.035\right)$. TPO-Ab, thyroid peroxidase antibody; PCOS, polycystic ovary syndrome.

Comparisons of the number of patients with EMT, PCOS among different age groups. In the present study, the 
numbers of patients with EMT and PCOS were compared among different age groups. The results showed that patients aged 28-35 years old took up the most in terms of EMT and PCOS (Fig. 1), among which the comparison of the percentage of positive simple TOP-Ab among the three age groups with EMT had no statistical difference $\left(\chi^{2}=1.005\right.$; $P=0.605$; Fig. 2$)$, while patients with PCOS aged 28-35 years showed relatively high percentage of positive simple TPO-Ab $\left(\chi^{2}=6.689\right.$; $\mathrm{P}=0.035$; Fig. 3).

\section{Discussion}

TPO is a thyroid-specific antigen, which destroys thyroid tissue mainly through infiltrating lymphocytes, thus resulting in autoimmune thyroid disease (AITD) (9). Currently, the correlation between AITD and recurrent miscarriage and pregnancy loss is confirmed in many studies (10-12), while the interference of thyroid autoantibodies in the normal fertility of women of childbearing age is still an outstanding problem (13-15). At present, there are relatively few studies on the influence of positive TPO-Ab on infertility. Therefore, a total of 529 infertile females were selected for experimental study so as to confirm the potential influence of positive TPO-Ab on infertility.

The results of the present study showed that basal hormone levels and BMI had no significant differences in females with positive simple TPO-Ab and normal thyroid function $(\mathrm{P}>0.05)$, while the percentages were relatively high in infertile females with positive simple TPO-Ab, and the expressions in EMT and PCOS were significant $(\mathrm{P}<0.05)$. A study of Chen et al $(15)$ showed that the incidence rate of positive TPO-Ab is high in infertile females, which is significantly related to idiopathic poor ovarian reserve function $(\mathrm{P}=0.020)$, leading to more unexplained infertility. A study of Medenica et al (16) showed that positive TPO-Ab is significantly correlated with EMT $(\mathrm{P}=0.001)$. A study conducted by Drobniak et al (17) showed that increased TPO-Ab is observed in $12-25 \%$ of females with autoimmune thyroid diseases, while the positive rate of TPO-Ab in females with PCOS and EMT is increased significantly, and TPO-Ab with a high titer is observed more frequently. A study of Sinha et al (18) showed that the positive rate of anti-TPO-Ab in patients with PCOS is increased $(\mathrm{P}<0.05)$, which is more significant in infertile patients with positive TPO-Ab than that in the control group (22.5\% vs. $1.25 \%, \mathrm{P}<0.01)$. Another study of Weghofer et al (19) also showed that the incidence rate of anti-TPO-Ab in patients with PCOS is significantly higher than that in the control group (normal females) $(\mathrm{P}<0.01)$. The aforementioned test results are basically identical with those of this study, which suggests that positive simple TPO-Ab may be correlated with female infertility caused by EMT and PCOS, while the mechanism of infertility of patients with EMT and PCOS caused by increased TPO-Ab still needs to be explored further.

There was no statistically significant difference in the incidence rate of EMT among the three age groups at different ages $(\mathrm{P}=0.605)$, while the positive rate of TPO-Ab in infertile females with PCOS aged 28-35 years old was increased significantly $(\mathrm{P}=0.035)$, which suggests that positive simple $\mathrm{TPO}-\mathrm{Ab}$ may have a greater impact on infertile females aged 28-35 years $(20,21)$.

This experimental study still has some shortcomings. The conditions of infertile females with positive TPO-Ab after treatment can be further analyzed to provide more support for this experimental study. As the experimental samples have regional limitations, a multi-center study on a large sample size can prove the significance of the experiment in a more powerful way.

In conclusion, positive simple TPO-Ab may be correlated with EMT and PCOS. Patients with EMT and PCOS (especially females with PCOS aged 28-35 years old) should undergo screening of thyroid autoantibodies actively to provide better interventions.

\section{Acknowledgements}

Not applicable.

\section{Funding}

No funding was received.

\section{Availability of data and materials}

The datasets used and/or analyzed during the present study are available from the corresponding author on reasonable request.

\section{Authors' contributions}

XW and XD conceived and designed the study. XX collected the patient data. FX and RF were responsible for the analysis and interpretation of the data. XW drafted this manuscript. $\mathrm{XD}$ revised the manuscript critically for important intellectual content. All authors read and approved the final manuscript.

\section{Ethics approval and consent to participate}

The present study was approved by the Ethics Committee of The Affiliated Wuxi Maternity and Child Health Care Hospital of Nanjing Medical University (Wuxi, China). Signed written informed consent was obtained from the patients and/or their guardians.

\section{Patient consent for publication}

Not applicable.

\section{Competing interests}

The authors declare that they have no competing interests.

\section{References}

1. Carp HJ, Selmi C and Shoenfeld Y: The autoimmune bases of infertility and pregnancy loss. J Autoimmun 38: J266-J274, 2012.

2. Deroux A, Dumestre-Perard C, Dunand-Faure C, Bouillet L and Hoffmann P: Female infertility and serum auto-antibodies: A systematic review. Clin Rev Allergy Immunol 53: 78-86, 2017.

3. Mobeen H, Afzal N and Kashif M: Polycystic ovary syndrome may be an autoimmune disorder. Scientifica (Cairo) 2016: 4071735, 2016. doi: 10.1155/2016/4071735.

4. Feldthusen AD, Pedersen PL, Larsen J, Toft Kristensen T, Ellervik $C$ and Kvetny J: Impaired fertility associated with subclinical hypothyroidism and thyroid autoimmunity: The Danish General Suburban Population Study. J Pregnancy 2015: 132718,2015 
5. Busnelli A, Paffoni A, Fedele L and Somigliana E: The impact of thyroid autoimmunity on IVF/ICSI outcome: A systematic review and meta-analysis. Hum Reprod Update 22: 793-794, 2016.

6. Al-Saab R and Haddad S: Detection of thyroid autoimmunity markers in euthyroid women with polycystic ovary syndrome: A case-control study from syria. Int J Endocrinol Metab 12: e17954, 2014

7. Zhong YP, Ying Y, Wu HT, Zhou CQ, Xu YW, Wang Q, Li J, Shen XT and Li J: Relationship between antithyroid antibody and pregnancy outcome following in vitro fertilization and embryo transfer. Int J Med Sci 9: 121-125, 2012.

8. Schankath AC, Fasching N, Urech-Ruh C, Hohl MK and Kubik-Huch RA: Hysterosalpingography in the workup of female infertility: Indications, technique and diagnostic findings. Insights Imaging 3: 475-483, 2012.

9. Unuane D, Velkeniers B, Anckaert E, Schiettecatte J, Tournaye H, Haentjens P and Poppe K: Thyroglobulin autoantibodies: Is there any added value in the detection of thyroid autoimmunity in women consulting for fertility treatment? Thyroid 23: 1022-1028, 2013.

10. Yoshioka W, Amino N, Ide A, Kang S, Kudo T, Nishihara E, Ito $\mathrm{M}$, Nakamura $\mathrm{H}$ and Miyauchi $\mathrm{A}$ : Thyroxine treatment may be useful for subclinical hypothyroidism in patients with female infertility. Endocr J 62: 87-92, 2015.

11. Speer G: The impact of thyroid function in women of reproductive age: Infertility, pregnancy and the postpartum period. Orv Hetil 154: 2017-2023, 2013 (In Hungarian).

12. Boufas D, Vryonidou A, Mastorakos G and Ilias I: Thyroid function and autoimmunity versus number of pregnancies. J Reprod Infertil 17: 240-242, 2016.

13. Plowden TC, Schisterman EF, Sjaarda LA, Zarek SM, Perkins NJ, Silver R, Galai N, DeCherney AH and Mumford SL: Subclinical hypothyroidism and thyroid autoimmunity are not associated with fecundity, pregnancy loss, or live birth. J Clin Endocrinol Metab 101: 2358-2365, 2016
14. Duran B, Ozlü T, Koc O, Essitken C and Topçuoğlu A: Relationship of thyroid hormone levels and thyroid autoantibodies with early pregnancy loss and infertility. J Obstet Gynaecol 33: 862-864, 2013.

15. Chen CW, Huang YL, Huang RL, Tzeng CR and Chen $\mathrm{CH}$ : Idiopathic low ovarian reserve is associated with more frequent positive thyroid peroxidase antibodies. Thyroid 27: 1194-1200, 2017.

16. Medenica S, Nedeljkovic O, Radojevic N, Stojkovic M, Trbojevic B and Pajovic B: Thyroid dysfunction and thyroid autoimmunity in euthyroid women in achieving fertility. Eur Rev Med Pharmacol Sci 19: 977-987, 2015.

17. Drobniak A, Kanecki K, Grymowicz M and Radowicki S: Serum leptin concentration in women of reproductive age with euthyroid autoimmune thyroiditis. Gynecol Endocrinol 32: 128-131, 2016.

18. Sinha U, Sinharay K, Saha S, Longkumer TA, Baul SN and Pal SK: Thyroid disorders in polycystic ovarian syndrome subjects: A tertiary hospital based cross-sectional study from Eastern India. Indian J Endocrinol Metab 17: 304-309, 2013.

19. Weghofer A, Himaya E, Kushnir VA, Barad DH and Gleicher N: The impact of thyroid function and thyroid autoimmunity on embryo quality in women with low functional ovarian reserve: A case-control study. Reprod Biol Endocrinol 13: 43, 2015.

20. Andreeva P: Thyroid gland and fertility. Akush Ginekol (Sofiia) 53: 18-23, 2014 (In Bulgarian).

21. Fumarola A, Grani G, Romanzi D, Del Sordo M, Bianchini M, Aragona A, Tranquilli D and Aragona C: Thyroid function in infertile patients undergoing assisted reproduction. Am J Reprod Immunol 70: 336-341, 2013.

This work is licensed under a Creative Commons Attribution-NonCommercial-NoDerivatives 4.0 International (CC BY-NC-ND 4.0) License. 